\title{
Condição corporal ao parto e perfil metabólico de cabras alpinas no início da lactação
}

\section{Larissa Pires Barbosa ${ }^{1 *}$, Marcelo Teixeira Rodrigues ${ }^{2}$, José Domingos Guimarães ${ }^{2}$, Vitor Valério Maffili ${ }^{3}$, Lincoln da Silva Amorim ${ }^{1}$, Américo Fróes Garcez Neto ${ }^{4}$}

\author{
${ }^{1}$ Programa de Pós-graduação em Zootecnia - Universidade Federal de Viçosa - UFV. \\ 2 Universidade Federal de Viçosa - UFV. \\ ${ }^{3}$ Centro de Pesquisa Gonçalo Moniz - FIOCRUZ. \\ 4 Universidade Federal do Vale do São Franscisco - UNIVASF.
}

RESUMO - Objetivou-se avaliar a influência da condição corporal ao parto sobre alguns metabólitos sanguíneos em cabras no início da lactação. As cabras (68 no total) foram distribuídas em três grupos de acordo com o escore da condição corporal (ECC) ao parto: entre 1,00 e 2,75 (animais magros); entre 2,75 e 3,50 (condição corporal intermediária); e entre 3,50 e 5,00 (animais gordos). O experimento foi realizado nos primeiros 60 dias de lactação e as coletas de sangue, após o parto e semanalmente até a oitava semana de lactação. Foram feitas análises para determinação das concentrações de ácidos graxos não-esterificados (AGNE), beta-hidroxibutirato (BHBA), glicose, colesterol total e lipoproteína de alta densidade (HDL). As concentrações plasmáticas dos metabólitos não diferiram entre os grupos. As concentrações de AGNE, nos animais dos três grupos, ao parto e nas oito primeiras semanas de lactação, foram de 658,46 e 232,90 mg/dL, respectivamente, e os valores de BHBA ao parto, de 10,31 $\pm 3,0 \mathrm{mg} / \mathrm{dL}$ para os animais magros; 6,88 $\pm 0,9 \mathrm{mg} / \mathrm{dL}$ para os intermediários; e 4,21 \pm 0,8 $\mathrm{mg} / \mathrm{dL}$ para os animais gordos. Os valores de glicose ao parto foram de 112,54 $\pm 15,4 \mathrm{mg} / \mathrm{dL}$ para os animais magros; 90,93 \pm 13,5 mg/dL para os animais intermediários; e 132,47 $\pm 26,7 \mathrm{mg} / \mathrm{dL}$ para os animais gordos. Os valores médios de colesterol total e de HDL nas oito primeiras semanas pós-parto foram de 109,01 $\pm 2,5$ e 54,9 $\pm 1,39 \mathrm{mg} / \mathrm{dL}$ para os animais magros; $83,0 \pm 21,7$ e $56,0 \pm 1,30 \mathrm{mg} / \mathrm{dL}$ para os intermediários; e 84,3 23,9 e 54,5 $\pm 1,93 \mathrm{mg} / \mathrm{dL}$ para os gordos. As alterações no metabolismo energético de cabras leiteiras de média produção diminuem, independentemente da condição corporal ao parto, quando fornecida dieta de alta qualidade e com altos níveis de energia.

Palavras-chave: caprino, condição corporal, perfil metabólico, pós-parto

\section{Body condition and metabolic profile of Alpine goats at the onset of lactation}

\begin{abstract}
An experiment was carried out to study the influence of the body condition score at birth on blood metabolites of goats in early lactation. Sixty-eight goats were placed in three groups according to the body condition score (BCS) at parturition: from 1.00 to 2.75 (thin animals); 2.75 and 3.50 (intermediate body condition) and from 3.50 to 5.00 (fat animals). The experimental was carried out in the first sixty days of lactation and blood sampling started after parturition at weekly intervals up to the eighth week of lactation. Analyses were made of the non-esterified fat acids (NEFA), betahydroxibutirate (BHBA), glucose, total cholesterol and high density lipoprotein (HDL). The plasma concentrations of the metabolites did not differ among the groups. The NEFA concentrations in the animals in the three groups at birth and in the first eight weeks of lactation were 658.46 and $232.90 \mathrm{mg} / \mathrm{dL}$, respectively and the values of BHBA at birth were 10.31 $\pm 3.0 \mathrm{mg} / \mathrm{dL}$ for the thin animals, $6.88 \pm 0.9 \mathrm{mg} / \mathrm{dL}$ for the intermediate animals and $4.21 \pm 0.8 \mathrm{mg} / \mathrm{dL}$ for the fat animals. The glucose values at birth were $112.54 \pm 15.4 \mathrm{mg} / \mathrm{dL}$ for the thin animals, $90.93 \pm 13.5 \mathrm{mg} / \mathrm{dL}$ for the intermediate animals and $132.47 \pm 26.7 \mathrm{mg} / \mathrm{dL}$ for the fat animals. The average of total cholesterol and HDL in the first eight weeks after birth were $109.01 \pm 2.5$; and $54.9 \pm 1.39 \mathrm{mg} / \mathrm{dL}$ for the thin animals, $83.0 \pm 21.7$ and $56.0 \pm 1.30 \mathrm{mg} / \mathrm{dL}$ for the intermediate animals and $84.3 \pm 23.9$ and $54.5 \pm 1.93 \mathrm{mg} / \mathrm{dL}$ for the fat animals. The changes in the energy metabolism of dairy goats with medium production decreased, regardless of the body condition at birth, when supplied with high quality feed with high energy levels.
\end{abstract}

Key Words: body condition, goats, metabolic profile, post-partum

Este artigo foi recebido em 4/7/2007 e aprovado em 6/11/2008.

Correspondências devem ser enviadas para: Ipires73@yahoo.com.br

*Endereço atual: Universidade Federal do Recôncavo da Bahia - UFRB. 


\section{Introdução}

No período de transição, três semanas finais da gestação e três semanas iniciais da lactação, a fêmea passa por profundas alterações endócrinas e metabólicas, ocorrendo queda nas concentrações plasmáticas de alguns hormônios e elevação de outros, como também aumento nas demandas metabólicas para o crescimento fetal e, posteriormente, para lactação (Grimard et al., 1995). Concomitantemente, ocorre redução no consumo de matéria seca, decorrente da compressão do rúmen pelo feto e da presença de hormônios envolvidos na homeorresia (Rodrigues et al., 2006).

A velocidade como estas mudanças ocorrem muitas vezes limita a capacidade de adaptação do animal em manter sua homeostase, predispondo a ocorrência de distúrbios periparturientes, como as doenças metabólicas, os problemas reprodutivos e a diminuição do desempenho lactacional (Grimard et al., 1995).

A mobilização das reservas corporais neste período, que resulta em redução do escore da condição corporal (ECC) e do peso corporal, é necessária para satisfazer as exigências de energia e proteína dos animais (Rodrigues et al., 2006).

Essa mobilização de reservas corporais vai levar a alterações fisiológicas, traduzidas em alterações endócrinas e metabólicas, podendo então ser avaliadas por meio de mensurações das concentrações plasmáticas de metabólitos sanguíneos. Um exemplo dessas alterações é a correlação altamente positiva entre as reservas de gordura e os níveis de ácidos graxos não-esterificados (AGNE) plasmático (Rueg et al., 1995), os quais aumentam próximo ao parto e podem preceder a redução no consumo de alimentos, além de outros constituintes metabólicos relacionados ao equilíbrio energético dos animais (Rodrigues et al., 2006).

O ECC ao final da gestação e os níveis de energia das dietas têm sido apontados como fatores determinantes do desempenho de animais em lactação (Rodrigues et al., 2006). Contudo, maiores informações são necessárias para determinar o ECC ideal ao parto em cabras leiteiras e nos vários estágios da lactação. Assim, realizou-se este estudo com o objetivo de avaliar as relações entre o ECC em cabras leiteiras ao parto e a resposta animal por meio de concentrações plasmáticas de alguns metabólitos sanguíneos.

\section{Material e Métodos}

O estudo foi realizado no Setor de Caprinocultura do Departamento de Zootecnia da Universidade Federal de Viçosa (UFV), em Viçosa, Minas Gerais, utilizando-se 68 cabras da raça Alpina, previamente selecionadas quanto ao escore da condição corporal, à ordem de parição (fêmeas de segunda e terceira lactações), à produção leiteira e à condição clínica.

Os animais foram confinados em baias individuais de piso ripado e com dimensão de 1,5 × 2,0 m e receberam a mesma dieta, composta de silagem de milho, farelo de soja (Glycine max L.), fubá de milho (Zea mays L.), calcário calcítico e sal mineral (Tabela 1), na forma de mistura completa, fornecida duas vezes ao dia (7h30 e 17 h). A ração foi calculada para atender às exigências nutricionais de cabras em lactação com produção até 5 kg de leite/dia, de acordo com recomendações apresentadas pelo AFRC (1998). Para que se permitisse consumo voluntário, uma quantidade adicional de $10 \%$ do consumo do dia anterior foi fornecida. As fêmeas foram ordenhadas manualmente, duas vezes ao dia (às 6 h30 e 16 h) dentro da própria baia.

Os animais foram distribuídos em três grupos, com base na avaliação do ECC ao parto, segundo Morand-Fehr \& Hervieur (1999), em escala de 1 a 5, com intervalos de 0,25 ponto, na qual o escore 1 correspondeu a cabras muito magras e o escore 5 , a cabras obesas, de modo que as cabras magras apresentaram ECC entre 1,00 e 2,75 (no total, 26 animais); as cabras com ECC intermediária, de 2,75 e até 3,50 (total de 27 animais); e as cabras gordas, de 3,50 até 5,00 (15 animais no total).

Coletas de sangue foram realizadas na veia jugular, para armazenamento do plasma e posterior análise dos metabólitos. Em todos os animais, a primeira coleta de sangue foi realizada imediatamente após o parto e depois semanalmente, até completarem oito semanas de lactação,

Tabela 1 - Composição da ração experimental (\% MS)

\begin{tabular}{lr}
\hline Item & $\% \mathrm{MS}$ \\
\hline Silagem de milho & 52,94 \\
Fubá de milho & 27,25 \\
Farelo de trigo & 11,03 \\
Farelo de glúten de milho & 5,00 \\
Ureia & 1,20 \\
Minerais & 2,58 \\
Composição nutricional & \\
Proteína bruta (\%) & 18,00 \\
Proteína degradável no rúmen (\%) & 11,50 \\
Proteína não-degradável no rúmen (\%) & 6,85 \\
Fibra em detergente neutro (\%) & 31,46 \\
Fibra em detergente ácido (\%) & 17,57 \\
Extrato etéreo (\%) & 2,60 \\
Carboidratos não-fibrosos (\%) & 38,00 \\
Lignina (\%) & 1,27 \\
Cinzas (\%) & 1,18 \\
Cálcio & 0,80 \\
Fósforo & 0,30 \\
Energia líquida (Mcal/kg de MS) ${ }^{1}$ & 1,70 \\
\hline 1 Estmada de acoro &
\end{tabular}

${ }^{1}$ Estimada de acordo com Moe \& Tyrrell (1975): EL = 0,0245*NDT-0,12 
sempre antes do fornecimento da dieta pela manhã. O sangue foi coletado em tubos vacuolizados contendo anticoagulante heparina e com antiglicolítico fluoreto de sódio, para análises de ácidos graxos não-esterificados (AGNE), $\beta$-hidroxi-butirato (BHBA), colesterol total, lipoproteína de alta densidade (HDL) e glicose. Imediatamente após a coleta, as amostras foram centrifugadas, durante 15 minutos a 336,3 $\mathrm{g}$, e divididas em alíquotas e armazenadas a $-20^{\circ} \mathrm{C}$ em tubetes com capacidade de $1,5 \mathrm{~mL}$. Após o período das coletas, as amostras armazenadas de plasma foram submetidas a análises laboratoriais.

A determinação da concentração plasmática de BHBA foi feita utilizando-se o kit $^{\circ}$ 310-A (Sigma Diagnostics ${ }^{\circledR}$ ), que se baseia no método enzimático e leitura por colorimetria, densidade ótica com comprimento de onda de $340 \mathrm{~nm}$, descrito por Willianson et al. (1962) (coeficiente de variação, $\mathrm{CV}=88,4 \%)$.

Para determinação da concentração plasmática de AGNE, utilizou-se o kit Wako, NEFAC (kitn ${ }^{\circ}$ 994-75409 $\left.{ }^{\circledR}\right)$, que se baseia no método enzimático e leitura pela colorimetria, densidade ótica com comprimento de onda de $560 \mathrm{~nm}$, segundo Johnson \& Peter (1993) (CV = 60,48\%).

A concentração plasmática de glicose foi determinada utilizando-se o kit n ${ }^{\circ}$ 15-100 (Sigma Diagnostics ${ }^{\circledR}$ ), que utiliza o método enzimático da glicose oxidase descrito por Tietz (1982) e leitura por meio de colorimetria (CV =23,91\%).

A concentração plasmática de colesterol total foi determinada utilizando o kit $^{\circ}{ }^{\circ} 01400$ (Laborlab), por meio do método enzimático da enzima colesterol oxidase com leitura por colorimetria ( $\mathrm{CV}=26,79 \%)$.

A concentração plasmática de HDL foi determinada utilizando-se o kit $\mathrm{n}^{\circ} 213$ (Analisa Diagnostica ${ }^{\circledR}$ ), por meio do método enzimático-Trinder com leitura por colorimetria $(\mathrm{CV}=20,70 \%)$. Todos os ensaios foram realizados em triplicatas, obtendo-se a média entre os resultados.

Os animais foram distribuídos em delineamento experimental inteiramente casualizado com três tratamentos, diferentes números de repetições (26 no grupo de animais magros; 27 no grupo dos animais intermediários; e 15 no grupo de animais gordos).

Na análise estatística foi utilizada análise de variância (SAEG 8.0) para verificar diferenças entre as concentrações de BHBA, AGNE, glicose, colesterol total e HDL e, para as variáveis significativas, as médias foram comparadas utilizando-se o teste de Student Newman-Keuls (SNK) a $5 \%$ de probabilidade. Os valores de ácidos graxos nãoesterificados foram determinados pelo procedimento Linear Response Plateau para ajustar modelos de regressão descontínua, partindo de uma regressão linear simples.

\section{Resultados e Discussão}

Não houve influência do ECC ao parto na concentração de AGNE $(\mathrm{P}>0,05)$ (Tabela 2). Os valores médios apresentados pelos animais ao parto e da primeira até a oitava semana de lactação foram de 658,46 e 232,90 mg/dL, respectivamente.

Esses valores são compatíveis com os encontrados na literatura para as espécies bovina e caprina (Borges et al., 2001; Rodrigues et al., 2007). A ausência de diferença na mobilização do tecido adiposo no início de lactação entre os animais com diferentes condições corporais ao parto pode estar relacionada à alta qualidade da dieta, rica em energia (1,7 Mcal de EL/kg de MS) consumida pelos animais neste período, que reduziu as alterações no metabolismo energético, independentemente da condição corporal dos animais.

Mesmo os animais do grupo com alta ECC, apresentando menores valores de consumo de matéria seca com base no peso vivo $(3,55 \pm 0,27 ; 3,29 \pm 0,28$ e $2,77 \pm 0,21$, para os grupos com baixo ECC, médio ECC e alto ECC, respectivamente) e peso metabólico $(9,67 \pm 0,75 ; 9,16 \pm 0,75$ e 7,91 \pm 0,61, para os grupos com baixo ECC, médio ECC e alto ECC, respectivamente), em relação aos animais do grupo com baixo ECC e médio ECC, essa diminuição do consumo não foi suficiente para promover diminuição no peso e no ECC desses animais, ao longo do período estudado, como também aumento significativo das concentrações plasmáticas de AGNE, justificado também pelo uso de uma dieta rica em energia.

A produção leiteira não foi um fator relevante para interferir na mobilização de reservas corporais dos animais nos diferentes grupos e não foi influenciada pelo ECC ao parto. Os animais apresentaram produção média de 3,41 $\pm 0,2 ; 3,44 \pm 0,2$ e 2,68 $\pm 0,1$, respectivamente, para os grupos com baixo ECC, médio ECC e alto ECC.

Santos (1996), trabalhando com bovinos, não encontrou efeito da ECC na concentração de AGNE e, somente quando as comparações foram feitas semanal-mente, os animais de maior ECC apresentaram maior concentração nas primeiras semanas de lactação, o que difere dos resultados encontrados neste experimento. Em cabras leiteiras no período periparturiente, Rodrigues et al. (2006) também não encontraram efeito da ECC ao parto sobre a concentração de AGNE nas oito semanas iniciais de lactação.

Os animais dos três grupos apresentaram comportamento semelhante, com concentração plasmática bem elevada ao parto, seguida de diminuição imediatamente após o parto, até uma concentração considerada fisiológica (Figura 1). 
Tabela 2 - Concentrações plasmáticas de ácidos graxos não-esterificados (AGNE), beta-hidroxibutirato (BHBA) e glicose em cabras leiteiras no início da lactação

\begin{tabular}{|c|c|c|c|}
\hline Semanas pós-parto & Baixo ECC (entre 1,0 e 2,75) & Médio ECC (entre 2,75 e 3,5) & Alto ECC (entre 3,5 e 5,0 ) \\
\hline & \multicolumn{3}{|c|}{ AGNE (mg/dL)* } \\
\hline Parto & $754,2 \pm 72,5$ & $713,4 \pm 65,7$ & $507,8 \pm 72,9$ \\
\hline 1 & $389,3 \pm 35,8$ & $379,0 \pm 37,8$ & $314,6 \pm 41,7$ \\
\hline 2 & $319,8 \pm 30,5$ & $285,7 \pm 43,6$ & $215,0 \pm 23,2$ \\
\hline 3 & $278,3 \pm 38,4$ & $232,7 \pm 24,4$ & $183,4 \pm 17,7$ \\
\hline 4 & $259,8 \pm 34,6$ & $251,3 \pm 36,3$ & $208,5 \pm 18,6$ \\
\hline 5 & $189,7 \pm 25,2$ & $215,6 \pm 26,8$ & $192,6 \pm 21,4$ \\
\hline 6 & $203,9 \pm 23,5$ & $212,5 \pm 29,5$ & $170,3 \pm 22,4$ \\
\hline 7 & $205,2 \pm 25,3$ & $203,7 \pm 26,6$ & $169,8 \pm 14,8$ \\
\hline \multirow[t]{2}{*}{8} & $205,6 \pm 29,1$ & $178,7 \pm 16,1$ & $123,4 \pm 14,8$ \\
\hline & \multicolumn{3}{|c|}{ BHBA $(\mathrm{mg} / \mathrm{dL})^{*}$} \\
\hline Parto & $10,31 \pm 3,0$ & $6,88 \pm 0,9$ & $4,21 \pm 0,8$ \\
\hline 1 & $6,58 \pm 1,5$ & $4,70 \pm 0,6$ & $2,64 \pm 0,5$ \\
\hline 2 & $5,58 \pm 1,3$ & $4,29 \pm 0,4$ & $2,54 \pm 0,5$ \\
\hline 3 & $4,33 \pm 1,5$ & $2,92 \pm 0,3$ & $2,95 \pm 0,5$ \\
\hline 4 & $4,15 \pm 0,7$ & $3,88 \pm 0,4$ & $3,06 \pm 0,5$ \\
\hline 5 & $2,17 \pm 0,2$ & $2,31 \pm 0,3$ & $2,78 \pm 0,4$ \\
\hline 6 & $2,95 \pm 0,4$ & $3,75 \pm 0,4$ & $2,56 \pm 0,3$ \\
\hline \multicolumn{4}{|c|}{ Glicose $(\mathrm{mg} / \mathrm{dL})^{*}$} \\
\hline Parto & $112,54 \pm 15,4$ & $90,93 \pm 13,5$ & $132,47 \pm 26,7$ \\
\hline 1 & $43,85 \pm 1,8$ & $47,15 \pm 1,9$ & $47,40 \pm 2,1$ \\
\hline 2 & $49,00 \pm 1,5$ & $47,07 \pm 1,9$ & $51,33 \pm 1,3$ \\
\hline 3 & $46,19 \pm 1,3$ & $49,81 \pm 1,7$ & $52,93 \pm 1,7$ \\
\hline 4 & $50,54 \pm 1,7$ & $50,33 \pm 2,0$ & $51,87 \pm 2,4$ \\
\hline 5 & $47,31 \pm 1,5$ & $53,11 \pm 1,7$ & $55,47 \pm 2,2$ \\
\hline 6 & $50,58 \pm 1,5$ & $50,85 \pm 1,6$ & $56,47 \pm 3,1$ \\
\hline 7 & $47,65 \pm 1,4$ & $54,00 \pm 1,7$ & $53,33 \pm 1,9$ \\
\hline 8 & $52,96 \pm 1,9$ & $51,11 \pm 1,8$ & $53,00 \pm 2,2$ \\
\hline
\end{tabular}

Não há diferença entre médias dos tratamentos pelo teste Newman Keuls a 5\% de probabilidade ( $\mathrm{P}>0,05)$.

*Média e erro-padrão da média.
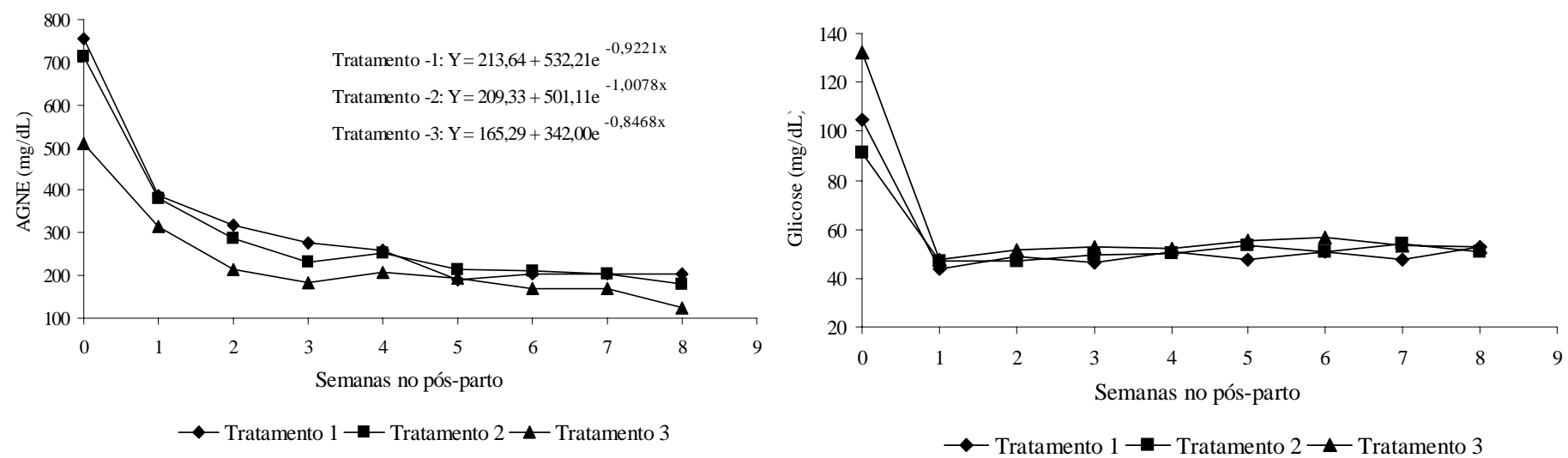

Figura 1 - Concentrações plasmáticas de ácidos graxos não-esterificados (AGNE) e glicose em cabras leiteiras com diversas condições corporais no período do parto até a oitava semana de lactação.

Por meio da análise de regressão (LRP), verifica-se que em média os animais apresentaram estabilização das concentrações de AGNE a partir do $10^{0}$ dia depois do parto, com concentrações de $220 \mathrm{mg} / \mathrm{dL}$, podendo-se sugerir então que essa concentração em caprinos seria considerada normal, consequência da mobilização fisiológica das reservas corporais.

Como a demanda energética do início da lactação normalmente não é suprida pela dieta, em decorrência da diminuição no consumo de matéria seca, predispondo 
os animais a entrar em balanço energético negativo, uma fonte alternativa deve estar disponível para a manutenção dos requerimentos do animal e, no caso dos ruminantes, a mobilização de triglicerídeos que é iniciada no pré-parto (Grummer, 1995) contribui com este aporte de energia.

Em bovinos a concentração plasmática de AGNE aumenta aproximadamente duas vezes entre os dias 17 e 2 antes do parto, apresentando um pico ao parto, devido a influências de vários hormônios lipolíticos que são liberados durante este período, como o glucagon e os corticoides, juntamente com a diminuição da insulina, hormônio lipogênico. Os níveis plasmáticos de AGNE decrescem rapidamente depois do parto e apresentam ainda concentrações mais elevadas que aqueles encontradas durante a gestação (Grummer, 1995). Essas alterações também são observadas na espécie caprina (Hussein \& Azab, 1998).

No período pós-parto, a taxa de lipólise sobrepõe a de lipogênese e disponibiliza maior quantidade de AGNE para fornecimento de energia aos tecidos periféricos. No fígado, o metabolismo dos AGNE depende da disponibilidade de glicose e de sua taxa de mobilização. Assim, podem ser completamente oxidados para produção de energia, parcialmente oxidados para produzir corpos cetônicos ou esterificados e estocados como triglicerídeos. O fígado dos ruminantes possui capacidade limitada para exportar triglicerídeos como lipoproteínas de muito baixa densidade (VLDL), de modo que a maior mobilização em relação à baixa exportação leva ao acúmulo hepático de gordura, e predispõe o animal aos distúrbios metabólicos (Head \& Gulay, 2001).

Uma relação inversa entre o consumo de alimento e as concentrações de AGNE plasmático tem sido demonstrada em muitos estudos (Bertics et al., 1992; Studer et al., 1993; Vazquez-Anon et al., 1994; Grummer, 1995) e está associada ao aumento da mobilização de tecido adiposo durante períodos de limitado consumo de energia (Pethick \& Dunshea, 1993).

Esse tipo de comportamento foi observado neste experimento, no qual as concentrações de AGNE foram bastante elevadas ao parto, com diminuição gradativa nas semanas subsequentes até valores fisiológicos, apresentando discreto aumento durante a semana do pico de lactação (quarta semana). O consumo, por sua vez, apresentou valores menores ao parto, elevando-se gradativamente nas semanas seguintes, com discreta diminuição na semana do pico de lactação (Figura 2).

Contudo, esse aumento da concentração de AGNE que começa no período pré-parto não é apenas consequência da redução do consumo voluntário de alimento. Estudos comprovam que o aumento plasmático de AGNE pode preceder essa redução do consumo, como demonstrado por Vazquez-Añon et al. (1994) verificaram que a elevação das concentrações plasmáticas de AGNE iniciou cinco dias antes do parto e que a diminuição do consumo só começou dois dias antes do parto. Esse resultado sugere que o consumo de matéria seca não é o único fator que influencia a mobilização do tecido adiposo, com consequente aumento da concentração plasmática de AGNE, que também envolve o sistema endócrino, como a ação dos hormônios lipolíticos nesse período (Santos, 1996).

Não houve influência da ECC ao parto na concentração de BHBA durante esse período ( $\mathrm{P}>0,05)$. Normalmente as alterações nas concentrações de BHBA acompanham as alterações de AGNE plasmático, assim, pela mesma razão dita anteriormente, a dieta reduziu o estresse no metabolismo energético dos animais, independentemente da ECC dos animais ao parto.

Além disso, os dados de BHBA comprovaram grande coeficiente de variação (CV $=88,4)$, principalmente os valores ao parto, o que dificultou observar diferenças entre os dados obtidos.

Dos 68 animais avaliados durante o período experimental, apenas cinco $(13,15 \%)$ apresentaram valores plasmáticos de BHBA acima de 10,4 mg/dL em pelo menos uma das semanas do experimento, o que caracteriza cetose subclínica (três animais) ou clínica (dois animais), todos pertenciam ao grupo de cabras com baixo ECC. Quando se retiraram esses cinco animais, o coeficiente de variação baixou para 60,8.

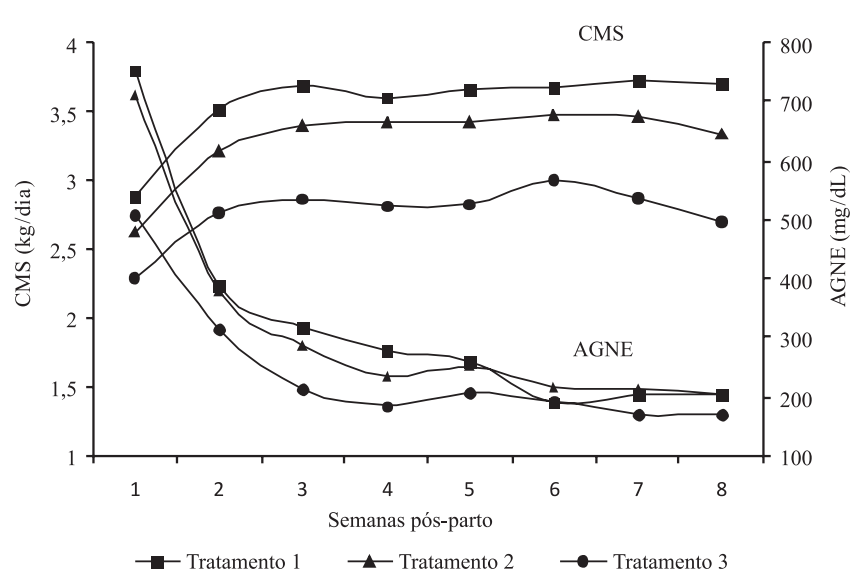

Figura 2 - Concentração plasmática de ácidos graxos nãoesterificados (AGNE) e consumo de matéria seca (CMS) em cabras leiteiras com diferentes condições corporais nas primeiras oito semanas no pós-parto. 
Os corpos cetônicos têm importante papel metabólico como poupador de glicose em ruminantes, por fornecerem acetil-CoA para ser oxidado pelos tecidos periféricos. Além disso, a glândula mamária utiliza o BHBA para síntese de gordura do leite (Lean et al., 1992). Os animais dos três grupos apresentaram o mesmo comportamento em relação às variações da concentração de BHBA durante o período pós-parto, onde se observam altos níveis durante o parto com diminuição gradativa até a oitava semana de lactação, chegando a concentrações normais, similar ao observado para as concentrações de AGNE.

As concentrações médias ao parto e da primeira até a oitava semana de lactação, incluindo os três grupos, foram de 7,13 e 3,08 mg/dL. Esses valores estão próximos àqueles encontrados por outros autores nas espécies bovina (Santos, 1996; Guedon et al., 1999) e caprina (Chang et al., 1996).

Em gado de corte, as concentrações plasmáticas de BHBA aumentam a partir do dia do parto, com valores entre 5 e $15 \mathrm{mg} / \mathrm{dL}$ (Borges et al., 2001) e valores de 10,4 mg/dL e são considerados o limite entre a normalidade e a cetose. Santos (1996) também encontrou aumento nas concentrações de BHBA do parto à terceira semana de lactação quando comparado com o período pré-parto em bovino de leite, encontrando valores de $3,7 \mathrm{mg} / \mathrm{dL}$ antes do parto e de $5,3 \mathrm{mg} / \mathrm{dL}$ no 14 으 dia do pós-parto.

As concentrações plasmáticas de glicose não diferiram $(\mathrm{P}>0,05)$ entre os animais dos três grupos (Tabela 1$)$. $\mathrm{O}$ valor médio da concentração de glicose plasmática ao parto e da primeira até a oitava semana de lactação foi de 111,98 e 50,55 mg/dL, respectivamente.

Klan \& Ludri (2002) demonstraram que as concentrações de glicose plasmática em cabras gestantes foram maiores que em cabras não-gestantes, exceto entre os dias 42 e 70 de gestação ( $59 \mathrm{mg} / \mathrm{mL})$. As cabras gestantes apresentaram níveis médios de $60 \mathrm{mg} / \mathrm{mL}$ entre os dias 42 e 56 de gestação e reduziram para 46 mg/mL entre os dias 112 e 126 no pré-parto.

Tanwar et al. (2000) estudando perfil bioquímico de cabras com cetose clínica, encontraram valores de glicose (mg/dL) de 33,46 $\pm 2,31$ para cabras com cetose e de 50,44 \pm 3,33 para cabras normais.

A concentração de glicose permanece estável ou pode sofrer leve aumento no período pré-parto. Um pico ocorre no parto e então decresce imediatamente no pós-parto, podendo apresentar concentrações um pouco abaixo do normal nas primeiras semanas, passando para concentrações normais e permanecendo estáveis, sem grandes flutuações, o que é justificado pelo seu grande controle homeostático (Vazquez-Anon et al., 1994; Guedon et al., 1999). O aumento no dia do parto (Figura 1) pode ser o resultado do aumento nas concentrações de glucagon, glicocorticoides e catecolaminas, que favorecem a gliconeogênese e a glicogenólise, promovendo a depleção dos estoques de glicogênio hepático (Grummer, 1995).

As primeiras alterações fisiológicas verificadas em animais com cetose são as reduzidas concentrações de glicose plasmáticas. Em condições normais, esta concentração é de $52 \mathrm{mg} / \mathrm{dL}$. Em vacas com cetose subclínica, a concentração média é de $46 \mathrm{mg} / \mathrm{dL}$ e, durante a cetose, é menor que $25 \mathrm{mg} / \mathrm{dL}$ de sangue (Herdt, 1988). As concentrações de glicose encontradas em vacas ao parto e aos 100 dias de lactação apresentaram valores médios de 90 e 60 mg/dL, respectivamente (Pires \& Fernandes, 1983). Todavia, Lindsay et al. (1993) sugeriram que a glicose plasmática pode não ser um bom indicador do estado energético, em decorrência da grande regulação homeostática a que está sujeita. Este eficiente controle homeostático da glicose pode explicar porque não houve efeito da ECC ao parto na concentração de glicose apresentada pelos animais deste experimento.

Apesar da maior demanda de glicose pela glândula mamária para síntese de lactose no início da lactação, os estoques de glicogênio hepático começam a se restabelecer e aumentam no $14^{0}$ dia no pós-parto. Isso provavelmente reflete o aumento da gliconeogênese hepática para suportar a lactação, que é função da maior concentração de corticosteroides próximo ao parto, evidenciando aumento na glicemia durante este período (Bell, 1995).

Não houve diferença $(P>0,05)$ entre os grupos de animais tratamentos para a concentração plasmática de colesterol total no período (Tabela 3). Os animais dos três grupos apresentaram padrão similar para o colesterol total, com concentrações menores ao parto, seguidas de aumento gradativo até os 60 dias no pós-parto (Figura 3); o valor médio geral para os animais, até a oitava semana de lactação foi de $110,84 \mathrm{mg} / \mathrm{dL}$.

Os valores do colesterol total estão dentro da variação encontrada na literatura para outros ruminantes, como bovinos. Na literatura, são relatados valores de $116 \mathrm{e}$ 109 mg/dL em vacas das raças Gir e Nelore. Ruas et al. (2000) verificaram menores valores do parto aos 45 dias, seguidos de aumento nas concentrações no transcorrer da lactação. Da mesma forma, Borges et al. (2001) trabalhando com animais das raças Gir e Nelore, encontraram valores médios de colesterol plasmático no pós-parto de $93 \mathrm{mg} / \mathrm{dL}$ e mencionaram que valores entre 80 e 260 mg/dL são normais para a espécie bovina.

O colesterol total reduz ao final da gestação, atinge o mínimo ao parto e aumenta até a nona semana pós-parto 
Tabela 3 - Concentrações plasmáticas de colesterol total e lipoproteínas de alta densidade (HDL) em cabras leiteiras do parto até a oitava semana de lactação

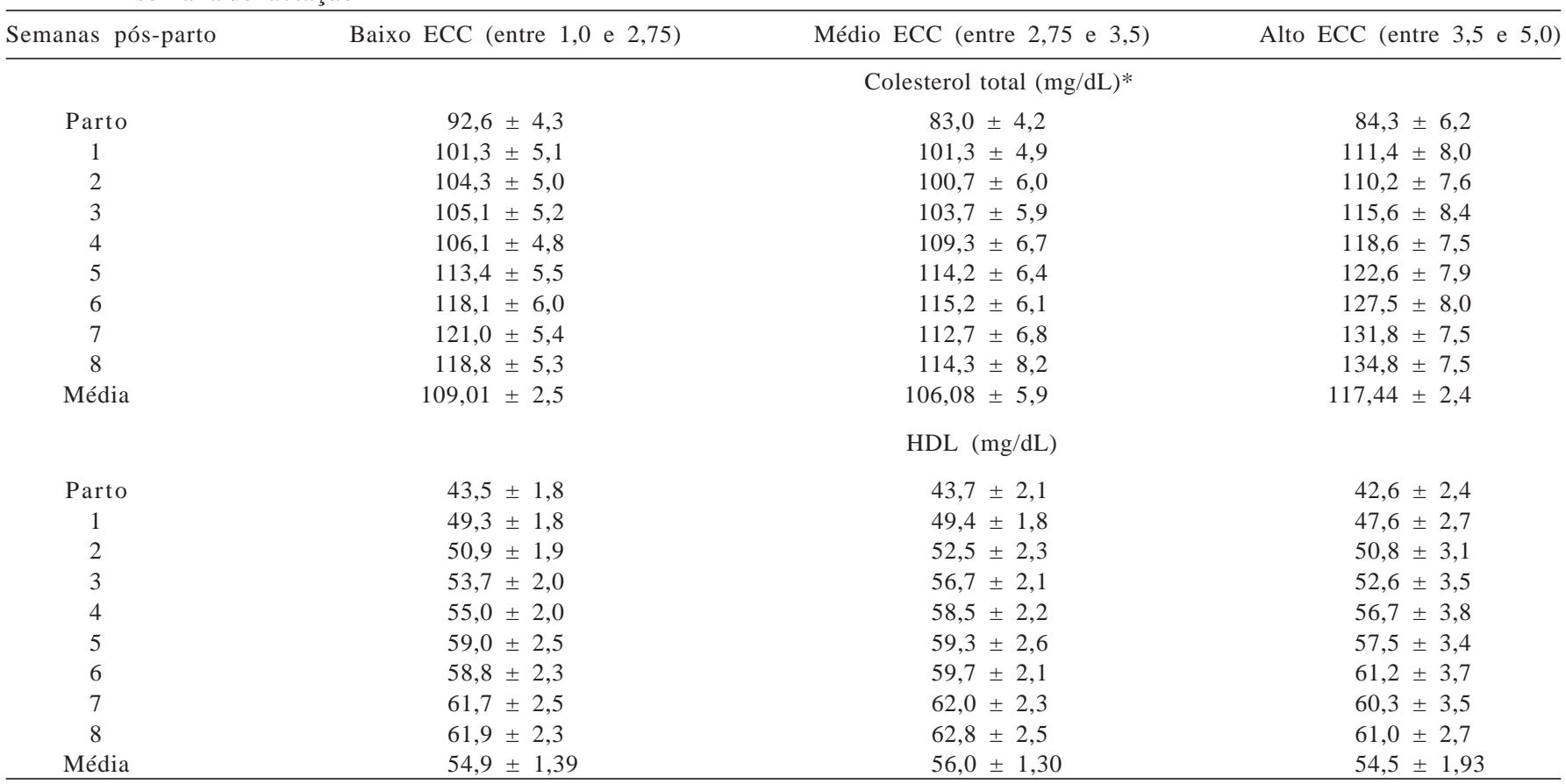

Não há diferença entre médias dos tratamentos pelo teste Newman Keuls, a 5\% de probabilidade de erro (P>0,05).

*Média e erro-padrão da média

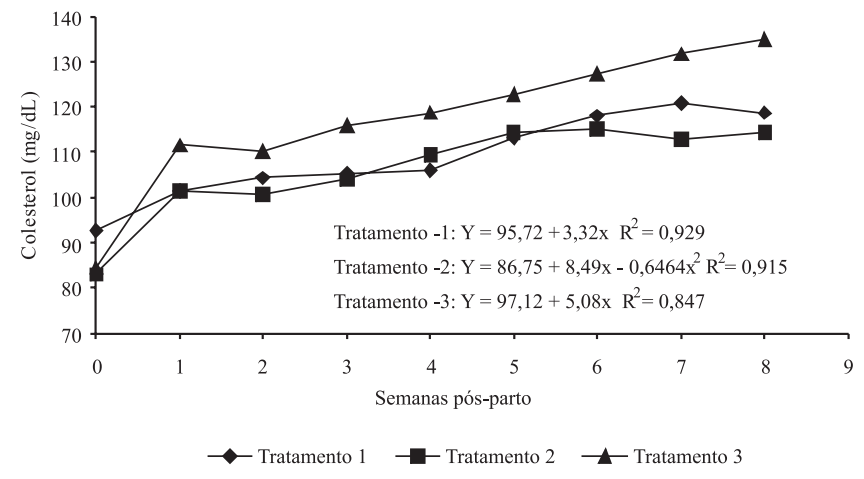

Figura 3 - Concentrações plasmáticas de colesterol total apresentadas por cabras leiteiras com baixo ECC, com médio ECC e com alto ECC durante o período pósparto.

em vacas de corte (Guedon et al., 1999). A menor concentração no pré-parto pode ser resultado de sua utilização pelo feto e, em menor extensão, pela utilização para a síntese de progesterona e de hormônios adrenais. Kappel et al. (1984) encontraram redução do colesterol antes do parto, com posterior aumento até os 88 dias de lactação em vacas da raça Holandesa. Este aumento do colesterol durante a lactação está associado com aumento na síntese de lipoproteínas e com mudanças entre os vários tipos de lipoproteínas, que são requeridas para o transporte de lipídeos. Desta forma, as concentrações plasmáticas de colesterol podem variar de acordo com a condição fisiológica do animal, a produção leiteira, o período da lactação e da dieta fornecida (González et al., 1996).

O colesterol total tem grande importância como precursor na produção dos hormônios esteroides e o colesterol total presente nas lipoproteínas HDL e LDL é o principal precursor desses hormônios, dessa forma pode influenciar os processos reprodutivos (O’Shaughnessy \& Wathes, 1985; Grummer \& Carrol, 1988).

Não houve diferença $(\mathrm{P}>0,05)$ entre os valores médios das concentrações plasmáticas de HDL entre os animais dos três grupos (Tabela 3). A concentração média apresentada pelos animais nas oito semanas de lactação foi de $55,17 \mathrm{mg} / \mathrm{dL}$.

Os animais dos três grupos apresentaram o mesmo comportamento no período citado, uma vez que houve menores concentrações de HDL ao parto, com aumento gradativo até a oitava semana no pós-parto, o que pode ser explicado da mesma forma que as variações apresentadas pelas concentrações de colesterol total plasmático.

\section{Conclusões}

O escore de condição corporal ao parto não influencia as concentrações plasmáticas de ácidos graxos nãoesterificados, $\beta$-hidroxibutirato, glicose, colesterol total e lipoproteína de alta densidade, principais metabólitos que caracterizam o metabolismo energético do animal. Portanto, para cabras leiteiras de média produção, entre 
2,5 a 3,0 kg/dia, independentemente da condição corporal ao parto, o fornecimento de uma dieta de alta qualidade e com altos níveis de energia pode reduzir as alterações no metabolismo energético.

\section{Agradecimentos}

À Universidade Federal de Viçosa (UFV) e ao Centro Nacional de Pesquisa (CNPq), pelo apoio financeiro na realização deste trabalho.

\section{Literatura Citada}

AGRICULTURAL AND FOOD RESEARCH COUNCIL - AFRC. Technical Committee on Responses to Nutrients. Report 10. The nutrition of goats. Nutrition Abstracts and Reviews (Series B), v.67, n.11, p.116, 1998.

BELL, A.W. Regulation of organic nutrient metabolism during transition from late pregnancy to early lactation. Journal Animal Science, v.72, p.2804-2819, 1995.

BERTICS, S.J.; GRUMMER, R.R.; CADORNIGA-VALINO, C. et al. Effect of prepartum dry matter intake on liver triglyceride concentration in early lactation. Journal of Dairy Science, v.75, p.1914, 1992.

BORGES, Á.M.; TORRES, C.A.A.; RUAS, J.R.M. et al. Concentrações plasmáticas de progesterona e metabólitos lipídicos em novilhas mestiças tratadas ou não com hormonio de crescimento e superovuladas. Revista Brasileira de Zootecnia, v.30, n.6, p.1689-1696, 2001.

CHANG, C.J.; CHEN, C.F.; WU, C.P. Changes in apparent mammary uptake of blood metabolites during involution uterine in dairy goats. Small Ruminant Research, v.24, p.49-54, 1996.

GRIMARD, B.; HUMBLOT, P.; THIBIER, M. Influence of postpartum energy restriction on energy status, plasma LH and estradiol secretion and follicular development in suckled beef cows. Journal Reproduction and Fertility, v.104, p.173-179, 1995.

GRUMMER, R.R. Impact of changes in organic nutrients metabolism on feeding the transition cow. Journal Animal Science, v.73, p.2820-2833, 1995.

GRUMMER, R.R.; CARROL, D.J. A review of lipoprotein cholesterol metabolism: importance to ovarian function. Journal of Animal Science, v.76, p.3882-3896, 1988.

GUEDON, L.; SAUMANDE, J.; DESBALS, B. Relationships between calf birth weight, prepartum concentrations of plasma energy metabolites and resumption of ovulation postpartum in limousine suckled beef cows. Theriogenology, v.52, p.779-789, 1999.

HEAD, H.H.; GULAY, M.S. Recentes avanços na nutrição de vacas no período de transição. In: SIMPÓSIO INTERNACIONAL DE BOVINOCULTURA DE LEITE: NOVOS CONCEITOS EM NUTRIÇÃO, 2001, Lavras. Anais... Lavras, 2001.

HERDT, T.H. Fuel homeostasis in the ruminant. Veterinary Clinics of North American. Food Animal Practice, v.4, p.213-232, 1988.

HUSSEIN, S.A.; AZAB, M.E. Plasma concentrations of lipids and lipoproteins in newborn kids and female Bladi goats during late pregnancy and onset of lactation. Dtsch Tierarztl Wochenschr, v.105, n.1, p.6-9, 1998.

JOHNSON M.M.; PETERS, J.P. Technical note: An improved method to quantify non-esterified fatty acids in bovine plasma. Journal of Animal Science, v.71, p.753-756, 1993.

KAPPEL, L.C.; INGRAHAM, R.H.; MORGAN, E.B. et al. Relationship between fertility and blood glucose and cholesterol concentrations in Holstein cows. Animal Journal Veterinary Research, v.45, n.12, p.2607-2612, 1984.
KHAN, J.R.; LUDRI, R.S. Changes in blood glucose, plasma nonesterified fatty acids and insulin in pregnant and non-pregnant goats. Tropical Animal Health and Production, v.34, n.1, p.81-90, 2002.

LEAN, I.J.; BRUSS, M.L.; BALDWIN, R.L. et al. Bovine ketosis: A review: II. Biochemistry and prevention. Veterinary Bull, v.62, n.1, p.1-14, 1992.

LINDSAY, D.R.; MARTIN, G.B., WILLIAMS, I.H. Nutrition and Reproduction. In: KING, G.J. (Ed.) Reproduction Domestic Animal, [S.I.: s.n.], 1993. p.459-491.

MOE, P.W.; TYRRELL, H.F. Symposium: production efficiency in the high producing cow. Efficiency of conversion of digested energy to milk. Journal of Dairy Science, v.58, p.602-610, 1975.

MORAND-FEHR, P.; HERVIEU, J. Apprécier l'éat corporel des chèvres: Intérêt et méthod. Reussir la Chevre, n.231, p.22-34, 1999.

O'SHAUGHNESSY, P., WATHES, D.C. Roles of lipoproteins and cholesterol synthesis in progesterone production by bovine luteal cells. Journal Reproduction and Fertility, v.74, p.425-432, 1985.

PETHICK, D.W.; DUNSHEA, F.R. Fat metabolism and turnover. In: FORBES, J.M.; FRANCE, J. (Eds.) Quantitative aspects of ruminant digestion and metabolism. Cambrige: $C A B$ International, 1993. p.291-311.

PIRES, C.C.; FERNANDES, L.C.O. Variação de peso e níveis plasmáticos de glicose, produção de leite e peso de terneiros durante o pós-parto de vacas de corte. Revista da Sociedade Brasileira de Zootecnia, v.12, n.1, p.60-71, 1983.

RODRIGUES, C.A.F.; RODRIGUES, M.T.; BRANCO, R.H. et al. Influência da condição corporal e da concentração de energia nas dietas no periparto sobre o desempenho de cabras em lactação. Revista Brasileira de Zootecnia, v.35, p.1560-1567, 2006.

RODRIGUES, C.A.F.; RODRIGUES, M.T.; BRANCO, R.H. et al Avaliação do consumo e de metabólitos plasmáticos de cabras gestantes, com duas condições corporais, alimentadas com dietas apresentando diferentes níveis de energia. Revista Brasileira de Zootecnia, v.36, p.945-952, 2007.

RUAS, J.R.M.; TORRES, C.A.A.; BORGES, L.E. et al. Efeito da Suplementação protéica a pasto sobre eficiência reprodutiva e concentrações sanguíneas de colesterol, glicose e uréia, em vacas Nelore. Revista Brasileira de Zootecnia, v.29, n.6, p.2043-2050, 2000.

RUEG, P.L.; MILTON, R.L. Body condition score of holstein cowon Prince Edward Island, Canada: relationships with yield, reproductive performance, and disease. Journal of Dairy Science, v.78, p.552-564, 1995.

SANTOS, J.E.P. Effect of degree of fatness prepartum on lactational performance and follicular development of early lactating dairy cows. 1996. 107f. Thesis (Master of Animal Science) - University of Arizona, Arizona, 1996.

STUDER, V.A.; GRUMMER, R.R.; BERTICS, S.J. Effect of prepartum propylene glycol administration on periparturient fatty liver in dairy cows. Journal of Dairy Science, v.76, p.2931-2939, 1993.

TANWAR, R.K.; TINNA, N.K.; GAHLOT, A.K. et al. Biochemical profile of clinical ketosis in goats. In: INTERNATIONAL CONFEREnCE ON GOATS, 7., 2000, Tolouse. Anais... Toulouse, 2000, p.306-307.

TIETZ, N.W. Fundamental of clinical chemistry. 2.ed. Philadelphia: Saunders, 1982. 240p.

VAZQUEZ-AÑON, M.S.; BERTICS, S.; LUCK, M. et al. Peripartum liver triclyceride and plasma metabolites in dairy cows. Journal of Dairy Science, v.77, p.1521-1528, 1994.

WILLIAMSON, D.H.; MELLANBY, J.; KREBS, H.A. Enzymatic determination of $\beta$-hidroxybutyric acid and acetoacetic acid in blood. Biochemical Journal, v.82, p.90-96, 1962. 